nuestros predecesores - y sobre todo, entender por qué lo dijeron, es decir, situarlos en su limitación histórica - no debe implicar una decadencia de pensamiento arqueológico. Como si, no teniendo otras cosas más novedosas que decir, nos refugiáramos en las formulaciones de los otros. No: este ejercicio de la critica es parte integrante de la historia.

Los temas aqui propuestos se dividen, al modo tradicional, en parcelas temáticas - escultura, arquitectura, urbanismo, cerámica, fuentes históricasprecedidas por un análisis historiográfico sobre la personalidad científica y la obra de Garcia y Bellido en el antiguo Rodrigo Caro ${ }^{19}$. Se trata asi de re-

Iv Estos son los ponentes y temas de la jornada cientifica que figuran en el programa: Javier Arce, "El Instituto español de Arqueologia Rodrigo Caro y la Revista Archivo Español de Arqueologia en la concepción de Antonio Garcia Bellidon; Martin Almagro, «De arquitectura ibérica»; Michael Blech, "La visión de la escultura ibérica, ayer y hoyn: Lorenzo Abad y Manuel Bendala, "La urbanistica del mundo ibérico: las nuevas perspectivas»; Fernando Quesada, "Los mercenarios ibéricos y la concepción histórica en la obra de Garcia y Bellidon: Ricardo Olmos, "Problemas historiográficos de cerámica ibérica e iconografian. Este último trabajo será publicado en Revista de estudios Ihericos, I. 1994. coger los principales aspectos que trató este autor en relación con el mundo ibérico. Su distribución responde pues, en gran medida, al viejo modelo de la investigación. Podrian, efectivamente, haber sido otras las propuestas pero creo que asi tenemos una repartición tradicional, sencilla y útil. Nuestro discurso $y$, sobre todo, la discusión que esperamos surja de todos los participantes servirán para entrelazar los aspectos parciales en la dialéctica de la globalidad.

Quedan, por último, unas breves palabras de acogida. Debo indicar que, siguiendo el espiritu de la tradición de estas jornadas, nos encontramos ante una reunión austera, sin la fluidez económica a que nos podian tener acostumbrados otros fastos cientificos. Por ello, en nombre del Departamento de Historia Antigua y Arqueologia del Centro de Estudios Históricos quiero agradecer muy cordialmente a todos - investigadores, familiares y amigos - su participación y su presencia. Especialmente quiero agradecer la colaboración desinteresada de los colegas que en estos dias han dedicado su tiempo y esfuerzo en preparar su comunicación y en ofrecernos hoy su valiosa aportación cientifica. Muchas gracias a todos.

\title{
GARCÍA Y BELLIDO Y EL INSTITUTO RODRIGO CARO
}

\author{
POR
}

\author{
JAVIER ARCE \\ Escuela Española de Historia y Arqueologia del C.S.I.C. Roma
}

En 1951 Antonio García y Bellido publicaba en Archivo Español de Arqueología una especie de manifiesto sobre lo que entendía él que deberia ser el recién creado Departamento o Instituto de Arqueología Rodrigo Caro. El motivo era, simplemente, que en ese año lo que era una sección del Instituto Diego Velázquez de Historia del Arte, se hizo independiente como Instituto de Prehistoria y Arqueología.

Considero este escrito de García Bellido el documento básico para comprender la idea que tenía sobre su Instituto y para entender en qué principios fundamentaba su acción y su futuro. En esta ocasión que se me brinda de recordar al gran investigador, arqueólogo e historiador, me limitaré a estudiar este texto que contiene todavía muchísimos puntos e ideas válidas, sugerencias y recomenda- ciones que invitan a la reflexión y que permite, sobre todo, contrastar lo que él se propuso y lo que hizo y el desarrollo posterior del Instituto.

Se plantea aquí una cuestión de principio, esto es, en qué medida y por qué hemos de seguir o continuar el espíritu del, llamémoslo así, «padre fundador", en qué medida debemos o no debemos considerar importante o necesaria la continuidad. Creo que podriamos estar de acuerdo en que mantener las líneas maestras y esenciales que han guiado e impulsado un centro de investigación o una revista, constituye la caracteristica definitoria y de identificación de la historia, la línea y la coherencia de este centro. Constituye su propia definición. En el curso del tiempo sobrevienen modificaciones y adaptaciones a los contextos y exigencias que van surgiendo; 
pero la revista Annales, por ejemplo, sigue siendo fiel al espiritu de sus fundadores - Braudel, Febvre, $y$ se identifica por ello. El Institute of Adranced Study de Princeton sigue manteniendo, con las aperturas y transformaciones lógicas. el espiritu que animó a sus fundadores y rigen alli los mismos modos de trabajo y comportamiento que cuando lo frecuentaban Panofsky o Kantorowicz. El Instituto Arqueológico Alemán, aún cuando ha tenido muchos y diversos directores, mantiene integro el espíritu que presidió la fundación de Istituto di Corrispondenza Archeologica. No se trata de no cambiar cuando haya que cambiar; se trata de cambiar sin alterar o transformar algunos componentes básicos. Permitanme pensar que ésta ha sido la clave del prestigio y competitividad de muchas instituciones cientificas europeas. Entre nosotros, es mucho más frecuente cambiarlo todo sin haber llegado siquiera aún a tener historia; reestructurar y suprimir, sin llegar casi nunca a hacer cuajar establemente algo.

Dicho esto, debo volver al texto de Garcia Bellido. Indicios del proceso de separación del Instituto Diego Velázquez de $\mathrm{H}^{\mathrm{a}}$ del Arte habian comenzado ya mucho antes de 1951. Si cuando el CSIC asumió el Centro de Estudios Históricos, después de la Guerra Civil, la Arqueología formaba con la $\mathrm{H}^{\mathrm{a}}$ del Arte el "Instituto Diego Velázquez», y su revista se denominaba Archivo Español de Arte y Arqueología (desde 1925), ya en 1940-1, ambas publicaciones estaban separadas en Archivo Español de Arte y Archivo Español de Arqueologia.

Una primera conclusión útil para eventuales olvidadizos: García Bellido entendia claramente que la Arqueología y la Historia del Arte deberian estar separadas como disciplinas independientes con objetivos diferentes y métodos también diferentes.

Pero el resultado de la separación implicó que el nuevo Instituto fuera de Arqueologia y Prehistoria. Esta denominación era, para García Bellido, un hecho transitorio: «Es evidente - señalaba en su manifiesto-que, pese a sus contactos y relaciones innegables, la Arqueologia Clásica y la Prehistoria son disciplinas fundamentalmente distintas. Es de esperar - concluia - que ambos busquen y hallen sus propios cauces en institutos hermanos, pero independientes").

Tenemos pues, que se hacian dos separaciones netas y claras: la Arqueologia, por un lado y la $\mathrm{H}^{\mathrm{a}}$ del Arte y la Prehistoria, separadas del mismo modo. Uno se puede preguntar: $i$ y para qué este afán de separar? ¿Se trata de una cuestión baladi, bizantina, inútil que pertenece a las clasificaciones de las ciencias del xIX, o del particularismo o, mejor, si se quiere, intención de ser el dueño de su parcela en plenas competencias sobre la misma? Puedo responder que no, en absoluto. Más adelante veremos que $\left({ }^{a} \mathrm{Belli}-\right.$ do tenia una idea precisa, amplia e interdisciplinar de "su» (entre comillas) departamento. Pero antes de pasar a examinar esta idea, es necesario responder a la pregunta del por qué esta separación entre Arqueologia, $\mathrm{H}^{\lrcorner}$del Arte y Prehistoria. La respuesta esta en la propia idea que Bellido tenia de la Arqueologia expresamente especificada por él mismo. A este propósito conviene no olvidar que «el manifiesto" de $G^{a}$ Bellido en el que delineaba las directrices del nuevo departamento, es del año 1951 y no olvidar que él mismo había experimentado en Europa - principalmente en Alemania - la experiencia de Departamentos de Arqueologia que le sirvieron, en mayor o menor medida, de modelos.

Para $G^{a}$ Bellido la Arqueología que el «R.Caro» debía hacer tenía el sentido de ser el estudio de las Antiguedades Griegas y Romanas, «incluyendo las de los pueblos sometidos directa o indirectamente a sus influjos». Ello excluia, en su opinión, «las otras arqueologias») - la americana, la egipcia, la mesopotámica que, señala expresamente - «aunque disciplinas afines por sus métodos, tienen otros objetos especificos y presuponen conocimientos auxiliares muy diversos (a los de la Arq. Clásica)».

Si aceptamos y entendemos la Arqueologia de esta forma, esto es, más que otra cosa la disciplina que estudia un periodo en un espacio y en un tiempo concretos, y no la entendemos como un método de análisis, la claridad de la división de $\mathrm{G}^{\mathrm{a}}$ Bellido es o resulta coherente y nitida. Por lo tanto, ni la $\mathrm{H}^{\mathrm{a}}$ del Arte ni la Prehistoria caben en ella más que de modo subsidiario. En definitiva para Bellido el elemento o factor que determina la separación son los conocimientos auxiliares o complementarios. Un historiador del Arte podria no saber griego - peor para él; un prehistoriador no por fuerza podría saber latín- de nuevo tanto peor. Pero un arqueólogo clásico, de las antigüedades griegas y romanas, no puede no saber ninguna de las dos lenguas. $\mathrm{Ni}$ tampoco podría ignorar la epigrafia, la numismática o el derecho antiguo: el prehistoriador no los necesita; el arqueólogo clásico, sí. Es como si pensaramos en un egiptólogo que ignòrase el demótico o el jeroglifico; o un estudioso de las culturas mesopotámicas que ignorase el cuneiforme.

Precisamente por ello, $\mathrm{G}^{\mathrm{a}}$ Bellido proponía , o mejor, aspiraba en su manifiesto a una «organización horizontal» de los Institutos de investigación humanística en el CSIC - son palabras suyas, que cito textualmente: "Cabe augurar que en un futuro próximo estos Institutos de Investigación se agrupen o asocien en sentido horizontal, incluso en la 
yuxtaposicion de sus laboratorios y bibliotecas. (reemos que llegará un dia en el que el Instituto de Arqueologia Rodrigo Caro, como el de Numismática y Epigrafía antiguas, se asocien estrechamente con su paralelo el «Nehrija», dedicado a la Filologia Clásica, conviviendo en una vecindad intima, más razonable que la que hasta ahora habia con el Instituto de Historia del Arte». Bellido entendia que este agrupamiento horizontal debia prevalecer sobre - cito textualmente - «institutos aislados acrónicos, heterogéneos, sin más lazo de unión que sus contactos verticales». Tengo que decir a este respecto que desde hace años he venido propugnando esta misma idea que he propuesto en diversos escritos al CSIC y a sus coordinadores. Pero parece ser que el mantenimiento de las parcelas de competencias están hoy más arraigadas y presentes que en el caso de $G^{a}$ Bellido. Porque en definitva - en mi opinión-su propuesta era razonable, lo razonable: un gran departamento de Ciencias de la Antiguedad que incluyese todas las disciplinas afines. Hoy que se habla tanto de interdisciplinariedad seguimos empeñados en una praxis compartimentada. Debo añadir que es obvio que la propuesta de $\mathrm{G}^{\mathrm{a}}$ Bellido sobre una biblioteca unificada con la de disciplinas afines cobra hoy una actualidad total.

En resumen: $G^{a}$ Bellido vió claramente que lo que él entendia por Arqueología - es decir la Arqueología Clásica , «se apoya en las Ciencias $\mathrm{Hu}$ manisticas» - son de nuevo palabras suyas. Y la Prehistoria en las "Ciencias Naturales». Esto sigue siendo completamente válido hoy.

Mi propuesta, en este punto, es ésta: las nuevas corrientes que han surgido recientemente en los estudios y análisis arqueológicos, la tecnificación de los mismos, los nuevos puntos de interés y problemáticas ciertamente fundamentales y que han significado un gran progreso en la Ciencia arqueológica, ¿no están más cerca de las «Natural Sciences»? Desde luego mi experiencia personal en la E.S.F. me confirma que ello es asi; y los proyectos de Prehistoria o de este tipo de arqueologias están conectadas con las «Natural Sciences» y no tanto con las llamadas "Humanities». Evidentemente unas se necesitan a otras; $y$ no son excluyentes, sino complementarias; o, como diria Bellido, subsidiarias. Pero poseen sus campos propios. Un reciente coloquio celebrado en Uppsala - por poner un ejemplo-viene a confirmar esta diversidad y complementariedad. Dedicado a "The Economies of Cult in the Ancient Greek World» viene a demostrar que los paradigmas técnicos o los modelos establecidos por algunos autores, no sirven literalmente para nada, si no hay un amplio, preciso y competente conocimiento y entendimiento tanto de la sociedad griega y sus instituciones, como de la religión. los textos (Isocrates, por ejemplo, o Demóstenes), la epigrafia. y los propios objetos en su contexto arqueológico, además de un buen conocimiento del funcionamiento de ciertas teorias económicas generales. Pero esto es, o seria, otro debate que quizás habria que hacer y nos aleja demasiado del tema de esta intervención.

El Instituto pensado por $\left(\mathrm{j}^{\mathrm{H}}\right.$ Bellido era, pues, en su definición primaria, un Instituto de Arqueologia Clásica separado de la Prehistoria, de la $\mathrm{H}^{\star}$ del Arte. pero yuxtapuesto a otras eventuales Ciencias de la Antigüedad. Su entendimiento de la amplitud espacial y temporal era también de gran elasticidad «el área mediterránea y sus aledaños" - señaló; y cronológicamente «rebasa los limites- escribe Bellido- tradicionales con que se suele poner fin a lo que se conoce por Edad Antigua, esto es, sus límites, en España, son el 711, el acontecimiento que cierra y completa el círculo de la cultura clásica», y la epoca carolingia, «que es para Europa central el verdadero limes cronológico entre lo antiguo y lo medieval». Esta amplitud de miras de $\mathrm{G}^{\mathrm{a}}$ Bellido demuestra ampliamente su correcto entendimiento de la dimensión del mundo clásico antiguo y está en plena consonancia y total acuerdo con los avances de la bibliografia más reciente y solvente - baste citar Peter Brown, R. Hodges o los trabajos de K. Randsborg sobre «el primer milenio».

La dimensión de las propuestas de Garcia Bellido y su concepción clara y rigurosa de su Departamento (un Departamento no "suyo», sino un departamento propio del Consejo Superior de Investigaciones Cientificas que forma parte por tanto de la Politica Cientifica nacional) no tuvo - por circunstancias que no es caso analizar aqui-su correspondencia esencial, o su contrapartida coherente: la creación de plazas de investigadores que pudieran afrontar y llenar de contenido la idea. El Rodrigo Caro se nutria de profesores universitarios que alternaban la docencia con la investigación de modo "saltuario" y el propio García Bellido, con su inmensa capacidad y amplios conocimientos, cubría casi todos los campos-arquitectura, epigrafia, historia militar, excavaciones, mosaicos, estatuaria, instrumentum domesticum, religión antigua, mundo ibérico, tartésicos, fenicio, céltico... Permitaseme una referencia personal a este respecto: cuando yo tuve la responsabilidad de la dirección del Departamento de Arqueología e $\mathrm{H}^{\mathrm{a}}$ Antigua dediqué, con mayor o menos éxito, todos mis esfuerzos a atraer y cubrir plazas en el Dpto. con una idea, que ha sido discutida por mis colegas e incluso calificada de errónea; la de encontrar especialistas competentes para los 
diversos aspectos que el Dpto. debia acoger dentro de la linea de la concepción de $\left(\mathrm{F}^{\mathrm{a}}\right.$ Bellido: desde el mundo celta hasta el mundo visigodo, desde la cerámica griega hasta la propia arqueologia de campo y espacial, desde la escultura romana (no olvidemos que la Prof. Pilar León sacó la plaza de colaboradora del Dpto. aunque luego optó por la universidad) hasta la incorporación de técnicas auxiliares de la Arqueologia (la Dra. Asunción Vila estuvo dos años con el Dpto. y se trasladó por fin a Barcelona). En torno a estos directores de investigación se podian ir formando grupos o núcleos en las diversas especialidades. Sigo pensando que el Departamento necesita el refuerzo romano y además un epigrafista.

Del espiritu del manifiesto de $\mathrm{G}^{\mathrm{a}}$ Bellido se deriva claramente una idea: la diversidad del campo de investigación del departamento y no la constante y unidireccional temática de los campos a cultivar.

En unas recientes declaraciones a la prensa, a propósito del Congreso Internacional de Arqueologia Clásica, el periodista destacaba - cómo nouna frase mía: «La Arqueología española es provinciana" frase que me ha ocasionado una marea de críticas y un enfado más que encendido por parte de muchos colegas. No he sido el primero en hacer esta declaración. Ya G ${ }^{\mathrm{a}}$ Bellido en 1951 en su manifiesto escribía en Archivo Español de Arqueología: «El signo que presidió hasta ahora nuestras actividades arqueológicas ha sido el del "provincialismo" científico con toda la limitación de horizontes y pobreza de espiritu que en si lleva el concepto. Nos interesaba "sólo" lo nuestro. Hay que hacer lo nuestro, sí. Pero creemos, subrayaba Bellido, que ha llegado el momento de empezar a cultivar una arqueología que no sea sólo nuestra, la provinciana ( palabra suya) sino la Arqueología patrimonial del Occidente..... debemos pues - proseguia - cortar amarras, dejar la navegación costera y lanzarnos a la alta mar de una Arqueologia sin adjetivos posesivos. Este es - concluia - ahora uno de los fines primordiales de nuestro Instituto con el que, sin abandonar lo nuestro se ha de procurar entrar en campos investigatorios hasta ahora inéditos para nosotros.»

En una estricta interpretación del texto, la finalidad primordial del Instituto de Arqueologia pensado por Bellido era la de hacer Arqueología clásica no local. Hasta qué punto ésto se haya cumplido, toca a la comunidad científica valorarlo. Lo que si puedo decir es que desde tiempo, pero especialmente ahora, el CSIC dispone de un punto de apoyo externo que resulta ser un complemento adecuado para que las aspiraciones de Bellido se cumplan: me refiero a la Escuela Española de $\mathrm{H}^{\mathrm{a}}$ y Arq. de Roma, centro y delegación del CSIC, dónde la formación de los becarios por fuerza rebasa el ámbito local y desde donde cualquier investigador dispone de posibilidades para ocuparse de la arqueologia que $\mathrm{Be}$ llido soñaba.

Consecuente con sus planteamientos $\mathrm{G}^{\mathrm{a}}$ Bellido, que dirigía también la revista Archivo Español de Arqueologia, entendió que ésta debía ser el órgano de expresión no sólo de los estudios sobre Hispania o la Peninsula Ibérica, sino que debian tener cabida en ella otros estudios no exclusivamente locales.

En este sentido Bellido era rotundo y claro en su manifiesto: «De todas las actividades creadoras del Instituto son éstas (las publicaciones) las más importantes. La publicación es «la tarea fundamental» (decia), la meta final, todo lo demás le está subordinado. Archivo Español de Arqueología ha de recoger fundamentalmente los estudios dedicados al mundo clásico (no olvidemos la extensión que para Bellido tenía este término) en todos sus aspectos». $\mathrm{Y}$ asi, la revista Archivo recoge desde 1951 articulos de autores españoles o extranjeros de una temática variada: Marchetti-Longhi sobre el teatro griego- desde el punto de vista arquitectónico, Max Wegner, H. G. Pflaum, G. Mansuelli, G. Alföldy, Mario del Chiaro, Claude Domergue, Beat Brenk, Tchernia, Schlunk y un largo etc. El Archivo de Bellido se articulaba como una revista cuyas características eran la variedad, la originalidad (de materiales y de interpretaciones), la extensión temática y cronológica (desde los fenicios hasta los visigodos) y el Noticiario. Era - es - una revista muy informativa de todo cuanto pudiere interesar a su público más frecuente - el español-y donde todo el mundo clásico y sus aledaños estaban presentes. Originalidad y novedad; calidad científica y variedad. La personalidad de Archivo no se basaba en los estados de la cuestión, ni en los artículos programáticos de método, sino en los resultados de la aplicación de esos métodos; no era una revista de debate, sino de resultados y tesis o propuestas de interpretación.

Para terminar, ¿qué resta de ese Instituto propuesto por $\mathrm{G}^{\mathrm{a}}$ Bellido? Son Vds. quienes deben responder a esta pregurtta. Yo sólo diré lo que hemos perdido en el camino o algunas cosas que hemos perdido en el camino. Hemos perdido el papel esencial que $\mathrm{G}^{\mathrm{a}}$ Bellido queria para el Instituto en la elaboración del CIL II (supplementum); hemos perdido la Hispania Antiqua Epigrafica; hemos perdido el Corpus Signorum Imperii Romani; hemos perdido la Biblioteca, en el sentido de espacio para el estudio y la consulta. Hemos hecho el Corpus de Mosaicos y la TIR avanza felizmente por citar sólo 
proyectos que Bellido tenia como propios del Instituto (luego, es cierto, han venido otros y hemos ganado otros); no sé si hemos perdido el archivo fotográfico. Hemos estado a punto de perder Archivo, lo que hubiera significado acabar con el Instituto, perderlo todo.

No es mi tarea aqui decir qué es lo que se debe hacer en el futuro; mi tarea ha sido recordar cómo nació y cuáles eran los fundamentos y las ideas guia con las que se creó el Dpto. de Arqueologia del ('SIC. Un Dpto. que el año 2001 cumplirá 50 años de historia, que habrá de celebrar adecuadamente, porque no debemos renunciar a la historia, una historia esforzada de todos sus muchos colaboradores, una historia, que a pesar de todo, creo que hace honor a su fundador, una historia de compromiso con la investigación de alta calidad y rigor que es la que practican cotidianamente sus miembros de todas las escalas.

\title{
LA URBANÍSTICA DEL MUNDO IBÉRICO. LAS NUEVAS PERSPECTIVAS
}

POR

\author{
L. ABAD y M. BENDALA \\ Universidad Autónoma de Madrid
}

Hace cincuenta anıs, el conocimiento que se tenía de la urbanística y la arquitectura ibérica era muy limitado y fragmentario. En palabras de D. Antonio García y Bellido, según escribia en los años cuarenta, «el número y nombre de las ciudades ibéricas conocidas por los textos antiguos era grande, sobre todo en Andalucia y Levante; pero desgraciadamente son poquísimas las que han llegado a nosotros aun en ruinas, y éstas son siempre, o casi siempre, ciudades que por su área, sus construcciones y el mismo anonimato con que se han dado a la luz, denuncian haber sido de rango relativamente bajo" (Ars Hispaniae I, Madrid, 1947, 203). Es la idea, expresada en un párrafo muy sintético, que desarrolla en trabajos de la época y algo posteriores (La Arquitectura entre los iberos, Madrid, 1945; Historia de España de R. M. Pidal, 1-3, Madrid, 1954, 373ss.), y corroboran los demás autores, entre ellos A. Arribas, en su conocida sintesis sobre Los Iberos, de unos años después (Barcelona, 1965): «Por desgracia, el conocimiento que tenemos de los núcleos de población ibéricos es muy limitado» (p. 117).

Era evidente un conocimiento muy parcial, y particularmente escaso o nulo para la zona de mediodía, la que según todos los indicios habia conocido un mayor desarrollo del urbanismo y el florecimiento de las ciudades de mayor rango. Se tenía en ello una consecuencia de la diferente intensidad de la investigación arqueológica en unas zonas y otras $y$, además, el resultado del diverso grado de continuidad histórica de los centros urbanos desde la Antiguedad a nuestros dias.

En cualquier caso, el despegue económico y cientifico a partir de los sesenta, que ha conducido a la multiplicación de los equipos cientificos y de las excavaciones, y la aplicación de una politica patrimonial más respetuosa con el legado arqueológico, permiten situarnos hoy ante un panorama completamente nuevo a la hora de contemplar el urbanismo ibérico.

El cambio cuantitativo (muchos más datos) y cualitativo (mejorregistro arqueológico, nuevos planteamientos metodológicos y teóricos) puede resumirse y plantearse en función de los dos planos principales en que se proyecta la ciudad; el conjunto de la organización urbana y las formas urbanisticas concretas de cada núcleo o tipo de centros. Dicho en otras palabras: lo urbano y lo urbanístico.

Se ha intensificado la investigación en busca de una aclaración definitiva a la cuestión de si el mundo ibérico alcanzó o no niveles urbanos. El resultado, con discusiones inevitables, es que el mundo ibérico es una cultura de nivel urbano, y la pregunta, en cada caso, no puede o no debe plantearse - a la altura de nuestro tiempo-en el sentido de si éste o aquel centro es una ciudad, sino si pertenece a una organización urbana y qué lugar y papel ocupa en ella. Se reconoce en el mundo ibérico una estruc- 